\title{
THE FULL MAPPING OF LOW-LYING EXCITED STATE RELAXATION DYNAMIC PATHWAYS FOR ACETOPHENONE
}

\begin{abstract}
ATTILA BENDE
ABSTRACT. Several relaxation pathways of the low-lying electronic excited state dynamics for acetophenone including also the singlet-triplet intersystem crossings were studied. The multireference Hartree-Fock and second order perturbation theory methods together with def2-tzvp basis set were used to characterize the equilibrium geometries and the crossing points between different potential energy surfaces (PES) up to the third excited state level considering both the singlet and triplet spin states. The electronic deactivation pathways studies reveal that the acetophenone shows several possible deactivation channels but their occurring probability strongly depends on the local profile of the PES of the intermediate states.
\end{abstract}

Keywords: acetophenone, conical intersection, intersystem crossing, spinorbit coupling, deactivation pathway

\section{INTRODUCTION}

Acetophenone $(\mathrm{C} 6 \mathrm{H} 5 \mathrm{C}(=\mathrm{O}) \mathrm{CH} 3, \mathrm{APN})$ is an aromatic ketone molecule with interesting photochemical and spectroscopic properties. Due to the electronic excitation of the $\pi$ electrons of the carbonyl group or the benzene ring, electron charges can easily migrate between the carbonyl fragment and the benzene ring and change the electronic structure of fragments. In general, this $\pi$ electron conjugation between the aromatic and carbonyl groups could influence the ordering of the $n \rightarrow \pi^{*}$ and $\pi \rightarrow \pi^{*}$ states, their photochemical reactivities, and the photo-dissociation mechanisms [1-3].

It was experimentally shown, that the gas phase absorption spectrum for APN in the region of 210-380 nm exhibits three broad peaks centered at 325,273 and $230 \mathrm{~nm}$, which are assigned to the electronic transitions from the ground state to S1, S2 and S3 excited states, respectively [4]. On

a Molecular and Biomolecular Physics Department, National Institute for Research and Development of Isotopic and Molecular Technologies, Str. Donat 67-103, C.P. 700, Cluj Napoca, RO-400293, Romania, attila.bende@itim-cj.ro 
the other hand, it is well known that APN shows both fluorescence [5] and phosphorescence [6] spectra; i.e., both the singlet and triplet states population can be experimentally achieved.

The first two singlet/triplet vertical and adiabatic excitation energies of the APN have already been studied [7-9] theoretically using complete active space self-consistent field (CASSCF) and second-order quasi-degenerate multi-reference perturbation theory (MR-PT2) methods. Theoretical studies on its non-radiative relaxation channel through the so-called conical intersections (Cls) [10] between different potential energy surfaces (PES) are rather limited, only Huix-Rotllant and co-workers [9] have studied in more details these crossing points. Most of the studies have been mainly focused on the mechanisms of photodissociation [11], especially, at S1/T2/T1 three-state intersection region $[1,12]$ or on the elucidation of the population mechanism of the triplet manifold $[9,13,14]$.

The aim of the present study is therefore to find, by means of minimum energy path and ISC point computations, alternative pathways for the acetophenone excited state relaxation mechanism. The paper is structured as follows: first, the computational details for the excited state characterization are discussed followed by a presentation and discussion of the conical intersection and intersystem crossing points as well as the spin-orbit couplings. Finally, conclusions about the excited state relaxation mechanism are given.

\section{RESULTS AND DISCUSSION}

Minima and crossing points. The ground state optimized geometry structure of APN was obtained using the SA-MCSCF method with the def2TZVP basis set, considering the $(10,9)$ complete active space configuration.

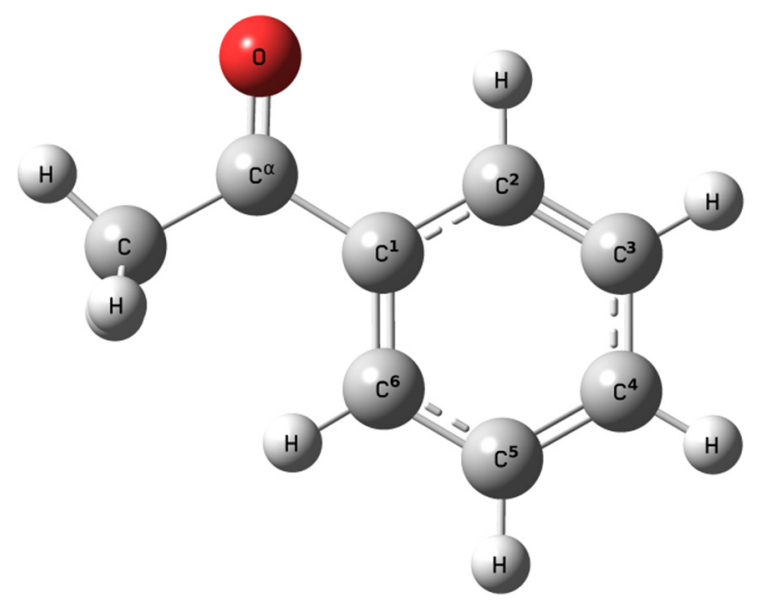

Figure 1. The equilibrium structure of the APN ground state geometry. 
The geometry of the APN molecules is shown in Figure 1. Based on the NPA charge analysis, the carbonyl fragment shows negative charge $(-0.024 e)$, while the benzene fragment contains the same amount of charge but with opposite sign. Concerning the benzene ring geometry, the $\mathrm{C}-\mathrm{C}$

Table 1. Internal bond coordinates (in $\AA$ ) of APN's optimized geometries at different equilibrium positions. ${ }^{a}$

\begin{tabular}{|l|cc|cccccc|cc|}
\hline Geom. & \multicolumn{2}{|c|}{ Carbonyl } & \multicolumn{9}{c|}{ Benzene } & \multicolumn{2}{c|}{$\begin{array}{c}\text { Energy } \\
\text { (in eV) } \\
\end{array}$} & $\mathrm{C}^{\alpha}=\mathrm{O}$ & $\mathrm{C}^{\alpha}-\mathrm{C}^{1}$ & $\mathrm{C}^{1}-\mathrm{C}^{2}$ & $\mathrm{C}^{2}-\mathrm{C}^{3}$ & $\mathrm{C}^{3}-\mathrm{C}^{4}$ & $\mathrm{C}^{4}-\mathrm{C}^{5}$ & $\mathrm{C}^{5}-\mathrm{C}^{6}$ & $\mathrm{C}^{6}-\mathrm{C}^{1}$ & $\mathrm{SCF}$ & $\mathrm{PT} 2$ \\
\hline$R_{e}\left(S_{0}\right)$ & 1.20 & 1.50 & 1.40 & 1.38 & 1.39 & 1.38 & 1.39 & 1.38 & - & - \\
$R_{e}^{C}\left(S_{1}\right)$ & 1.39 & 1.41 & 1.41 & 1.37 & 1.39 & 1.39 & 1.37 & 1.42 & 3.34 & 3.77 \\
$R_{e}^{B}\left(S_{1}\right)$ & 1.19 & 1.47 & 1.44 & 1.43 & 1.42 & 1.42 & 1.43 & 1.44 & 4.54 & 5.31 \\
$R_{T S}\left(S_{1}\right)$ & 1.24 & 1.46 & 1.44 & 1.43 & 1.42 & 1.42 & 1.43 & 1.44 & 4.64 & - \\
$R_{e}^{C}\left(S_{2}\right)$ & 1.44 & 1.35 & 1.47 & 1.40 & 1.36 & 1.45 & 1.33 & 1.45 & 6.05 & 6.45 \\
$R_{e}^{B}\left(S_{2}\right)$ & 1.22 & 1.44 & 1.46 & 1.45 & 1.40 & 1.37 & 1.42 & 1.46 & 5.28 & 4.78 \\
$R_{e}\left(S_{3}\right)$ & 1.36 & 1.39 & 1.51 & 1.43 & 1.34 & 1.45 & 1.41 & 1.40 & 6.30 & \\
$R_{e}^{C}\left(T_{0}\right)$ & 1.35 & 1.41 & 1.41 & 1.39 & 1.39 & 1.40 & 1.37 & 1.42 & 3.04 & 3.73 \\
$R_{e}^{B}\left(T_{1}\right)$ & 1.20 & 1.46 & 1.45 & 1.42 & 1.42 & 1.40 & 1.44 & 1.43 & 4.52 & 5.23 \\
$R_{e}^{B}\left(T_{2}\right)$ & 1.19 & 1.48 & 1.43 & 1.44 & 1.42 & 1.41 & 1.43 & 1.43 & 4.60 & 5.30 \\
\hline
\end{tabular}

${ }^{a}$ Bond lengths are given in $\AA$ and energies in $\mathrm{eV}$. The labelling of atoms is given in Figure 1.

bonds vary between 1.38 and $1.40 \AA$, showing a slight deviation from its value in the perfect aromatic ring. The vertical excitation energies as well as the equilibrium geometries of the first and second excited states calculated at Hartree-Fock (HF) were already done in our previous work [8]. Accordingly, the vertical excitation energies are $4.52 \mathrm{eV}$ for the $\mathrm{S} 1$ and 5.60 $\mathrm{eV}$ in case of $\mathrm{S} 2$ state, respectively. The corresponding values obtained at the XMS-CASPT2 level of theory are 4.54 and $4.85 \mathrm{eV}$, respectively. If one analyzes the charge redistribution for different excited states induced by the vertical excitation one can observe that the $S_{0} \rightarrow S_{1}$ transition induces $0.095 \mathrm{e}$ fractional electronic charge migration from the carbonyl fragment to the benzene group, while the $S_{0} \rightarrow S_{2}$ transition relocate -0.087 e charges from the benzene group to the carbonyl fragment. Once the AP molecule is excited and gets into the corresponding excited electronic state, it tries to reach its global (or in some circumstances the local) energy minimum. Since, the structures of the optimized geometries for the first and second excited singlet states were already presented in our previous work [8]; we show their internal bond values only in tabular form (see Table 1) without discussing them again in more detail. Considering the two molecular fragments of the carbonyl group and the benzene ring, one global and one 
local energy minimum were found for both $S_{1}$ and $S_{2}$ excited state cases. The only difference between the minima is the localization of the changes in the geometry of the fragments. For the simplicity, the geometries will be denoted by $R_{e}^{X}\left(Y_{i}\right)$, where $\mathrm{X}=\mathrm{B}$ or $\mathrm{C}(\mathrm{B}=$ benzene, $\mathrm{C}=$ carbonyl) is used to differentiate stationary points on carbonyl or benzene, $Y=S$ or $T$ is used to define the singlet or triplet spin state, $i=0,1$ or 2 is used to show the order of the given excited state related to the energy quantum number, while e indicates the nature of the stationary point (equilibrium geometry, $\mathrm{Cl}$, ISC or TS). Accordingly, the global energy minimum of the $\mathrm{S}_{1}$ state induces geo-metry changes on the carbonyl group $\left(R_{e}^{C}\left(S_{1}\right)\right.$ with $\left(\mathrm{n}, \Pi^{*}\right)$ excitation character), while the $S_{2}$ state's global minimum shows enlarged benzene ring's periphery $\left(R_{e}^{B}\left(S_{2}\right)\right.$ with $\left(\pi, \pi^{*}\right)$ excitation character). The opposite is true for the geometry changes for the local energy minima. Besides the analysis of the electronic configuration and geometry structure of the first two excited states, we have included in our present study the examination of the energy level of the $S_{3}$ third excited state. The vertical excitation energy of this electronic state is $6.98 \mathrm{eV}(177 \mathrm{~nm})$ obtained at SA-MCSCF/def2-TZVP levels of theory. The $S_{0} \rightarrow S_{3}$ vertical transition induces $0.190 \mathrm{e}$ fractional electronic charge migration from the benzene group to the carbonyl fragment and shows a very large oscillator strength $(f=0.0740)$.

Performing geometry optimization, we have observed important fragment deformations on both carbonyl and benzene parts (See Figure 2) where the $C^{\alpha}=O$ and the $C^{1}-C^{2}$ bonds significantly enlarge, while $C^{\alpha}-C^{1}$ one becomes much shorter. Since we are also interested in the electronic relaxation process over the triplet states, we have performed further geometry optimizations for the ground and for the first two triplet states. In this case, only the global energy minima are presented.

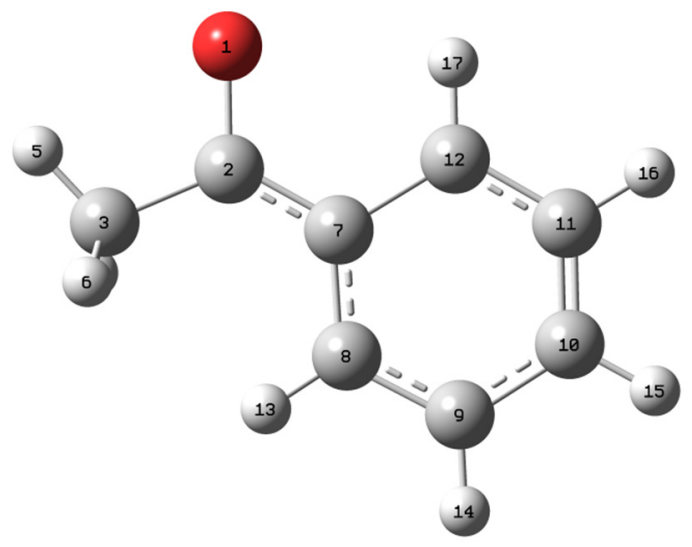

Figure 2. The equilibrium structure of the APN $S_{3}$ geometry. 
By comparing the geometry configurations of the singlet ground state and the minima of the triplet states, one can observe that the ground triplet state $\left(R_{e}^{C}\left(T_{0}\right)\right)$ geometry implies changes on the carbonyl group, while the first and second excited triplet states $\left(R_{e}^{B}\left(T_{1}\right)\right.$ and $\left.R_{e}^{B}\left(T_{2}\right)\right)$ show changes mainly on the benzene ring (See Table 1). It can be also observed that $R_{e}^{B}\left(T_{1}\right)$ and $R_{e}^{B}\left(T_{2}\right)$ global energy minima are close to each other both from energetic and geometry parameter points of view. One should also mention that, all the obtained minimum-energy structures have planar geometry configuration allowing the maximum delocalization of the $\pi^{*}$ orbitals along the benzene ring and the $\mathrm{C}=\mathrm{O}$ bond of the carbonyl fragment.

Table 2. Internal bond coordinates (in $\AA$ ) of APN's geometries at different crossing points (conical intersection or intersystem crossing). ${ }^{a}$

\begin{tabular}{|c|cc|cccccc|cc|}
\hline Geom. & \multicolumn{2}{|c|}{ Carbonyl } & \multicolumn{9}{|c|}{ Benzene } & \multicolumn{2}{c|}{$\begin{array}{c}\text { Energy } \\
\text { (in eV) }\end{array}$} \\
& $\mathrm{C}^{\alpha}=\mathrm{O}$ & $\mathrm{C}^{\alpha}-\mathrm{C}^{1}$ & $\mathrm{C}^{1}-\mathrm{C}^{2}$ & $\mathrm{C}^{2}-\mathrm{C}^{3}$ & $\mathrm{C}^{3}-\mathrm{C}^{4}$ & $\mathrm{C}^{4}-\mathrm{C}^{5}$ & $\mathrm{C}^{5}-\mathrm{C}^{6}$ & $\mathrm{C}^{6}-\mathrm{C}^{1}$ & SCF & $\mathrm{PT} 2$ \\
\hline$\left(S_{1} \otimes S_{2}\right)^{a}$ & 1.16 & 1.50 & 1.49 & 1.50 & 1.42 & 1.36 & 1.44 & 1.49 & 5.58 & 5.54 \\
$\left(T_{0} \otimes T_{1}\right)$ & 1.29 & 1.39 & 1.47 & 1.37 & 1.41 & 1.45 & 1.33 & 1.46 & 3.40 & 3.79 \\
$\left(T_{1} \otimes T_{2}\right)$ & 1.19 & 1.49 & 1.44 & 1.44 & 1.46 & 1.39 & 1.43 & 1.41 & 4.63 & 5.15 \\
$\left(T_{2} \otimes T_{3}\right)$ & 1.21 & 1.45 & 1.44 & 1.42 & 1.39 & 1.38 & 1.43 & 1.45 & 4.87 & 4.70 \\
$\left(S_{1} \otimes T_{1}\right)$ & 1.20 & 1.46 & 1.45 & 1.42 & 1.41 & 1.41 & 1.44 & 1.43 & 4.53 & 5.14 \\
$\left(S_{1} \otimes T_{2}\right)$ & 1.22 & 1.45 & 1.44 & 1.37 & 1.41 & 1.44 & 1.46 & 1.45 & 5.13 & 5.27 \\
$\left(S_{2} \otimes T_{3}\right)$ & 1.22 & 1.45 & 1.45 & 1.45 & 1.44 & 1.41 & 1.37 & 1.44 & 5.30 & 4.89 \\
\hline
\end{tabular}

aBond lengths are given in $\AA$ and energies in eV. The labelling of atoms is given in Figure 1.

The existence of crossing points between different PESs in the configuration space is the key of the fast radiationless decay of the electronic excited state relaxation [10]. Accordingly, we have continued our investigation by searching for different $\mathrm{Cls}$ between electronic states with similar spin configuration as well as for ISCs between electronic states with singlet and triplet spin configurations (See Table 2). As a first step, let to consider the crossing between the ground and the first excited electronic singlet spin states. Here, we found three different geometry configurations (See Tables 2 and 3) with relatively close energy values: $5.17 \mathrm{eV}, 5.26 \mathrm{eV}$ and $5.15 \mathrm{eV}$, respectively. The first two $\mathrm{Cl}$ structures show a broken benzene's ring planarity, close to the half-boat conformation found in the case of the isolated benzene molecule by Robb and co-workers $[15,16]$, while the third one presents only carbonyl group distortion where the $\mathrm{C}=\mathrm{O}$ bond enlarge over the single bond character (1.72 $\AA$ ). No any structure with planar benzene ring was found for this $S_{0} \otimes S_{1}$ crossing. Following the same 
idea, we have investigated several possible $\mathrm{Cl}$ configurations for the $\mathrm{S}_{1} \otimes \mathrm{S}_{2}$ crossing. For this case, four different geometry structures were found having three different structural characteristics: planar benzene ring, halfboat benzene ring and carbonyl group deformations.

Among them the most relevant is that one which shows the planar ring deformation (See Figure 3 and for its geometrical parameter Table 2) because its energy $(5.58 \mathrm{eV})$ lies very close to the $S_{2}$ vertical excitation energy value. The other three structures (See Table 4) show much higher energy values $(7.12 \mathrm{eV}, 7.91 \mathrm{eV}$ and $8.61 \mathrm{eV}$, respectively) and they are

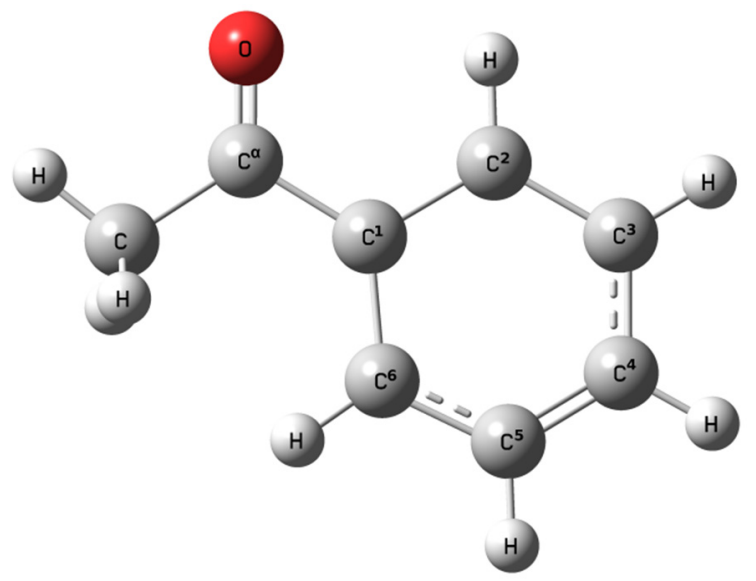

Figure 3. The equilibrium structure of the APN $\left(S_{1} \otimes S_{2}\right)^{\text {a }}$ geometry.

Table 3. Geometries of three different conical intersection configurations between the first excited $\left(S_{1}\right)$ and the ground $\left(S_{0}\right)$ singlet spin states.

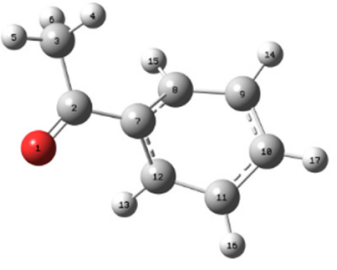

$\left(S_{0} \otimes S_{1}\right)^{a}$

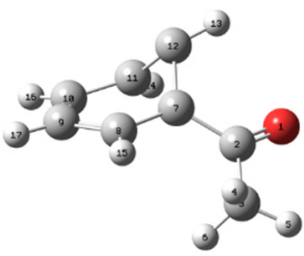

$\left(S_{0} \otimes S_{1}\right)^{b}$

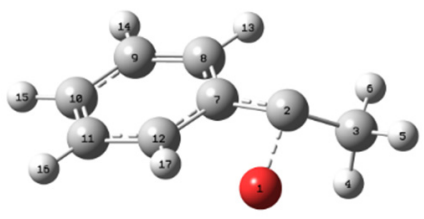

$\left(S_{0} \otimes S_{1}\right)^{c}$

\begin{tabular}{llllllllll}
\hline & $\mathrm{O}_{1}-\mathrm{C}_{2}$ & $\mathrm{C}_{2}-\mathrm{C}_{3}$ & $\mathrm{C}_{2}-\mathrm{C}_{7}$ & $\mathrm{C}_{7-\mathrm{C}_{8}}$ & $\mathrm{C}_{8}-\mathrm{C}_{9}$ & $\mathrm{C}_{9}-\mathrm{C}_{10}$ & $\mathrm{C}_{10}-\mathrm{C}_{11}$ & $\mathrm{C}_{11-\mathrm{C}_{12}}$ & $\mathrm{C}_{12}-\mathrm{C}_{7}$ \\
\hline$\left(S_{0} \otimes S_{1}\right)^{a}$ & 1.193 & 1.510 & 1.464 & 1.443 & 1.461 & 1.389 & 1.389 & 1.460 & 1.436 \\
$\left(S_{0} \otimes S_{1}\right)^{b}$ & 1.190 & 1.511 & 1.488 & 1.470 & 1.385 & 1.391 & 1.455 & 1.448 & 1.459 \\
$\left(S_{0} \otimes S_{1}\right)^{c}$ & 1.720 & 1.483 & 1.418 & 1.419 & 1.353 & 1.416 & 1.357 & 1.405 & 1.392 \\
\hline
\end{tabular}


Table 4. Geometries of three different conical intersection configurations between the second $\left(S_{2}\right)$ and the first $\left(S_{1}\right)$ excited singlet spin states.

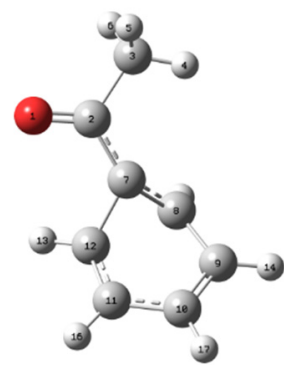

$\left(S_{1} \otimes S_{2}\right)^{b}$

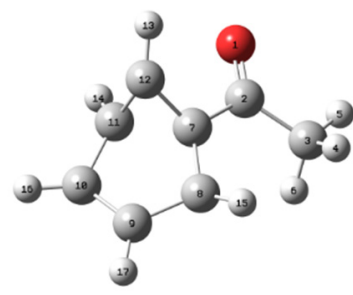

$\left(S_{1} \otimes S_{2}\right)^{c}$

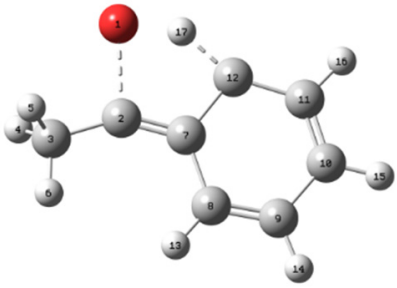

$\left(S_{1} \otimes S_{2}\right)^{d}$

\begin{tabular}{llllllllll}
\hline & $\mathrm{O}_{1}-\mathrm{C}_{2}$ & $\mathrm{C}_{2}-\mathrm{C}_{3}$ & $\mathrm{C}_{2}-\mathrm{C}_{7}$ & $\mathrm{C}_{7}-\mathrm{C}_{8}$ & $\mathrm{C}_{8}-\mathrm{C}_{9}$ & $\mathrm{C}_{9}-\mathrm{C}_{10}$ & $\mathrm{C}_{10}-\mathrm{C}_{11}$ & $\mathrm{C}_{11}-\mathrm{C}_{12}$ & $\mathrm{C}_{12}-\mathrm{C}_{7}$ \\
\hline$\left(S_{1} \otimes S_{2}\right)^{b}$ & 1.205 & 1.517 & 1.416 & 1.425 & 1.454 & 1.382 & 1.426 & 1.388 & 1.551 \\
$\left(S_{1} \otimes S_{2}\right)^{c}$ & 1.189 & 1.507 & 1.499 & 1.489 & 1.467 & 1.469 & 1.485 & 1.465 & 1.484 \\
$\left(S_{1} \otimes S_{2}\right)^{d}$ & 1.886 & 1.455 & 1.302 & 1.479 & 1.337 & 1.475 & 1.330 & 1.458 & 1.508 \\
\hline
\end{tabular}

slightly probable to be reached during the relaxation. From the phosphorescence point of view, the electronic relaxation over the triplet spin states also looks important. Starting from the $T_{0}$ ground triplet state its crossing with the $T_{1}$ first excited triplet state is reached at two different geometry configurations. The geometry with the lowest energy value $(3.40 \mathrm{eV})$ shows a planar configuration with enlarged $\mathrm{C}=\mathrm{O}$ bond (See Table 2 and Table 5), while the second one has a non-planar geometry $(4.93 \mathrm{eV})$ with benzene ring in half-boat form (See Table 5). Continuing the present investigation by moving one step up to the crossing between $T_{1}$ and $T_{2}$ states, yet another $\mathrm{Cl}$ point was found. This geometry also shows a planar configuration, with an enlarged benzene ring while the carbonyl group remains unchanged (See for its geometrical parameters Table 2 and Table 6). Moving even further, a new crossing point with similar geometry configuration was found between the $T_{2}$ and $T_{3}$ states. Its geometrical parameters are given in Table 2 and its molecular graphics is presented in Table 6 . In this case we found also for the carbonyl group small changes in the $\mathrm{C}^{\alpha}=\mathrm{O}$ and $\mathrm{C}^{\alpha}-\mathrm{C}^{1}$ bond distances.

To switch between different singlet and triplet states one need to locate the so-called intersystem crossing points and to compute the spin-orbit coupling to see how efficient could be the spin transition. Accordingly, we have searched for spin crossovers between $S_{1} \otimes T_{1}, S_{1} \otimes T_{2}, S_{2} \otimes T_{2}$ and $S_{2} \otimes T_{3}$ electronic spin states. Apart from the case of $S_{2} \otimes T_{2}$, we have successfully located these 
Table 5. Geometries of two different conical intersection configurations between the ground $\left(T_{0}\right)$ and the first $\left(T_{1}\right)$ excited triplet spin states.

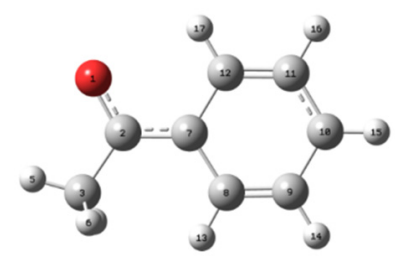

$\left(T_{0} \otimes T_{1}\right)^{a}$

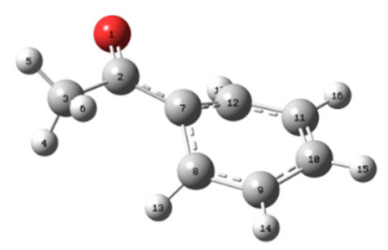

$\left(T_{0} \otimes T_{1}\right)^{b}$

\begin{tabular}{llllllllll}
\hline & $\mathrm{O}_{1}-\mathrm{C}_{2}$ & $\mathrm{C}_{2}-\mathrm{C}_{3}$ & $\mathrm{C}_{2}-\mathrm{C}_{7}$ & $\mathrm{C}_{7}-\mathrm{C}_{8}$ & $\mathrm{C}_{8}-\mathrm{C}_{9}$ & $\mathrm{C}_{9}-\mathrm{C}_{10}$ & $\mathrm{C}_{10}-\mathrm{C}_{11}$ & $\mathrm{C}_{11-}-\mathrm{C}_{12}$ & $\mathrm{C}_{12}-\mathrm{C}_{7}$ \\
\hline$\left(T_{0} \otimes T_{1}\right)^{a}$ & 1.293 & 1.510 & 1.390 & 1.462 & 1.332 & 1.448 & 1.410 & 1.369 & 1.469 \\
$\left(T_{0} \otimes T_{1}\right)^{b}$ & 1.203 & 1.512 & 1.443 & 1.434 & 1.446 & 1.386 & 1.384 & 1.446 & 1.428 \\
\hline
\end{tabular}

Table 6. Geometries of conical intersection configurations between different excited triplet spin states.

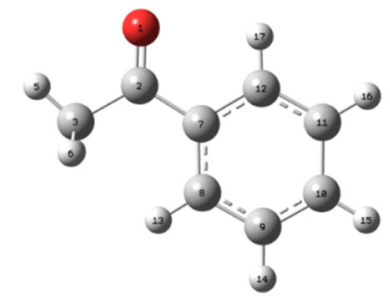

$\left(T_{1} \otimes T_{2}\right)$

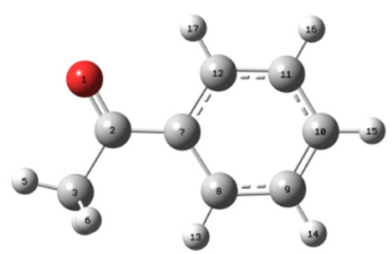

$\left(T_{2} \otimes T_{3}\right)$

\begin{tabular}{llllllllll}
\hline & $\mathrm{O}_{1}-\mathrm{C}_{2}$ & $\mathrm{C}_{2}-\mathrm{C}_{3}$ & $\mathrm{C}_{2}-\mathrm{C}_{7}$ & $\mathrm{C}_{7}-\mathrm{C}_{8}$ & $\mathrm{C}_{8}-\mathrm{C}_{9}$ & $\mathrm{C}_{9}-\mathrm{C}_{10}$ & $\mathrm{C}_{10}-\mathrm{C}_{11}$ & $\mathrm{C}_{11-\mathrm{C}_{12}}$ & $\mathrm{C}_{12}-\mathrm{C}_{7}$ \\
\hline$\left(T_{1} \otimes T_{2}\right)$ & 1.190 & 1.511 & 1.492 & 1.414 & 1.426 & 1.390 & 1.461 & 1.438 & 1.439 \\
$\left(T_{2} \otimes T_{3}\right)$ & 1.213 & 1.516 & 1.448 & 1.449 & 1.428 & 1.384 & 1.388 & 1.424 & 1.443 \\
\hline
\end{tabular}

crossing points between PESs. The first geometry we discuss is the $S_{1} \otimes T_{1}$ crossing point. This geometry is characterized mainly by the benzene ring planar deformation, where $C^{1}-C^{2}, C^{5}-C^{6}$ and $C^{6}-C^{1}$ are slightly stretched, while the carbonyl fragment's geometry remains almost unchanged. Its geometrical parameters are given in Table 2 and its molecular graphics is presented in Table 7. Analyzing the strength of the spin-orbit coupling, we have observed that there is no any zero-field splitting, namely, the triplet spin state does not separate in three sublevels and the energies of the lowest $0^{\text {th }}$-order and of the lowest SO states derived from the Breit-Pauli Hamiltonian are identical. On the other hand, we found that the coupling between the singlet and triplet spin states is quite strong; the magnitude of 
the spin-orbit coupling is $19.18 \mathrm{~cm}^{-1}$. Continuing our investigation, we have found that the $S_{1}$ singlet state can cross not only the $T_{1}$ triplet state but also the $T_{2}$ one. The geometry of this ISC point only slightly differs from the previous case of the $S_{1} \otimes T_{1}$ crossing. In this later case, the benzene ring planar deformations will be a bit larger (For the geometrical parameters see Table 2 and for its molecular graphics see Table 7) as well as we observe a small elongation of the $C^{\alpha}=O$ bond. Again, we have not found any zero-field splitting, and the magnitude of the spin-orbit coupling is only $1.15 \mathrm{~cm}^{-1}$. As we already mention, we have been unable to find any crossing point between the $S_{2}$ singlet and $T_{2}$ triplet states, so we proceed to analyze the $\mathrm{S}_{2} \otimes \mathrm{T} 3$ crossing case. The geometry of this structure is almost identical with the previously obtained $S_{1} \otimes T_{2}$ geometry, the only difference is the relative rotation with 180 degrees of the benzene ring with respect to the carbonyl group. The geometrical parameters of the $S_{2} \otimes T_{3}$ ISC point are given in Table 2, while its molecular graphics is presented in Table 7.

Table 7. Geometries of three different intersystem crossing configurations between the excited singlet and triplet spin states.

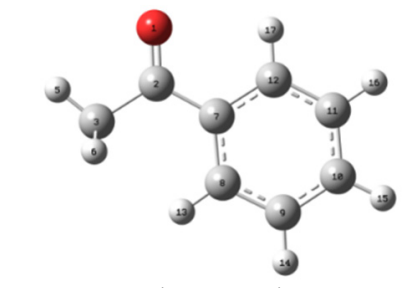

$\left(S_{1} \otimes T_{1}\right)$

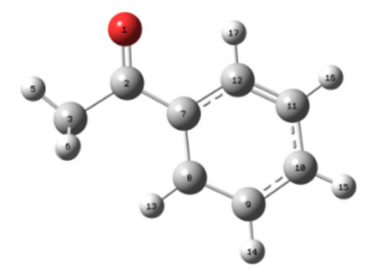

$\left(S_{1} \otimes T_{2}\right)$

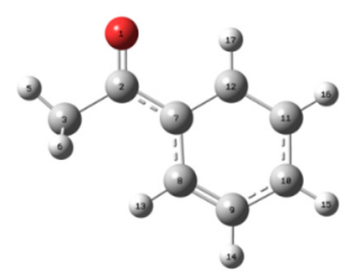

$\left(S_{2} \otimes T_{3}\right)$

\begin{tabular}{llllllllll}
\hline & $\mathrm{O}_{1}-\mathrm{C}_{2}$ & $\mathrm{C}_{2}-\mathrm{C}_{3}$ & $\mathrm{C}_{2}-\mathrm{C}_{7}$ & $\mathrm{C}_{7}-\mathrm{C}_{8}$ & $\mathrm{C}_{8}-\mathrm{C}_{9}$ & $\mathrm{C}_{9}-\mathrm{C}_{10}$ & $\mathrm{C}_{10}-\mathrm{C}_{11}$ & $\mathrm{C}_{11}-\mathrm{C}_{12}$ & $\mathrm{C}_{12}-\mathrm{C}_{7}$ \\
\hline$\left(S_{1} \otimes T_{1}\right)$ & 1.197 & 1.514 & 1.464 & 1.432 & 1.436 & 1.405 & 1.414 & 1.422 & 1.446 \\
$\left(S_{1} \otimes T_{2}\right)$ & 1.216 & 1.513 & 1.452 & 1.450 & 1.457 & 1.437 & 1.407 & 1.367 & 1.437 \\
$\left(S_{2} \otimes T_{3}\right)$ & 1.216 & 1.515 & 1.446 & 1.441 & 1.366 & 1.409 & 1.437 & 1.454 & 1.453 \\
\hline
\end{tabular}

The spin-orbit coupling is only $0.32 \mathrm{~cm}^{-1}$ which is even smaller than that of in the previous $S_{1} \otimes T_{2}$ case and does not show any zero-field splitting.

Relaxation pathways. As we have already known $[8,9]$ the oscillator strength of the $S_{0} \rightarrow S_{1}$ electronic transition is very small. Therefore, one needs to excite the APN molecule to its higher electronic states in order to have efficient radiation absorption. Considering the next electronic transition of $\mathrm{S}_{0} \rightarrow \mathrm{S}_{2}$ it looks that this is already sufficient to find our molecular system in an excited state $(f=0.0091)$. The nature of this electronic excitation is a 
$(\pi, \pi) \rightarrow\left(\pi, \pi^{*}\right)$ orbital transition. Once the molecule will reach its second excited state level, it begins its electronic excited state relaxation. It has two possibilities: either changing its electronic state to $S_{1}$ through the $S_{1} \otimes S_{2} \mathrm{Cl}$ crossing or moving towards to its $S_{2}$ equilibrium conformation. From the optimized geometry position of the $S_{2}$ state one has again two further possibilities. First: to reach the previous $\mathrm{Cl}$ crossing and move further the $S_{1}$ state and second: to change its spin configuration to the triplet one via the $\mathrm{S}_{2} \otimes \mathrm{T}_{3}$ ISC point. Energetically, both alternatives are feasible, but considering the weak SO coupling $\left(0.32 \mathrm{~cm}^{-1}\right)$ between the singlet and the triplet states, the first option looks more achievable than the second one. Hereupon the system can rapidly relax to its $S_{1}$ electronic state, since all these geometry changes involve benzene ring's planar deformation which doesn't mean significant energetic expense. As soon as, the molecule has changed its excited state, it reaches the local energy minimum on the $R_{e}^{B}\left(S_{1}\right)$ PES. Energetically, this geometry is a meta-stable conformation; since there is another minimum with lower energy $\left(R_{e}^{C}\left(S_{1}\right)\right)$ showing geometrical changes at the carbonyl fragment. This $R_{e}^{B}\left(S_{1}\right)$ local minimum we consider to be the "cornerstone" of the further relaxation steps. From this point, the molecule can easily undergo to its $R_{e}^{C}\left(S_{1}\right)$ global minimum by passing a $0.1 \mathrm{eV}$ higher $R_{T S}\left(S_{1}\right)$ saddle point (For geometrical parameters see Table 1, for its molecular graphics see Figure $4 a$ and for its potential energy curve see Figure $4 b)$. After reaching the global energy minimum of the $S_{1}$ state we have been unable to find any other critical point ( $\mathrm{Cl}$ or ISC) which would have moved the system to its singlet ground or a triplet state. The final step of the relaxation can be made only via the fluorescence deactivation [5]. Another very likely direction would be the crossing from the singlet spin state to the triplet one through the $S_{1} \otimes T_{1}$ ISC point. This is close both energetically and geometrically to the local minimum geometry and the spin-orbit coupling is also strong enough to perform the spin transition. Once the molecule has changed its spin configuration the relaxation decay continues on the triplet deactivation path. Namely, from the $R_{e}^{B}\left(T_{1}\right)$ configuration the molecule comes rapidly down to its ground triplet state through the $\left(T_{0} \otimes T_{1}\right)$ a crossing. Finally, the molecule loses its triplet state and turn back to its ground singlet state by a long-life radiation (phosphorescence) deactivation. For the whole relaxation pathway along the triplet spin state see Figure 5 . Besides of these two highly probable relaxation pathways which finally leads to different radiation emissions, APN can also show non-radiative deactivation. Indeed, close to the $R_{e}^{B}\left(S_{1}\right)$ local energy minimum there is a $\mathrm{Cl}$ crossing $\left(\mathrm{S}_{0} \otimes \mathrm{S}_{1}\right)$ which could drive the molecule from the first excited to its ground state. 


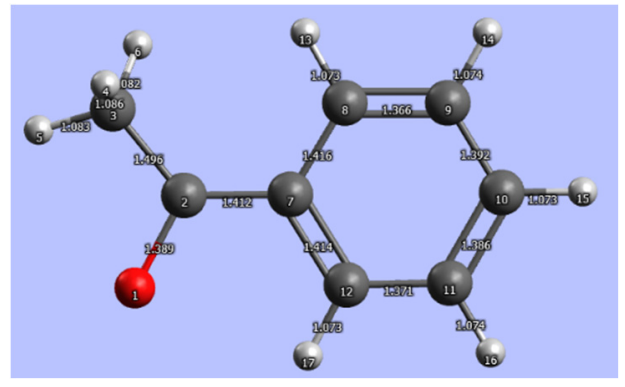

(a)

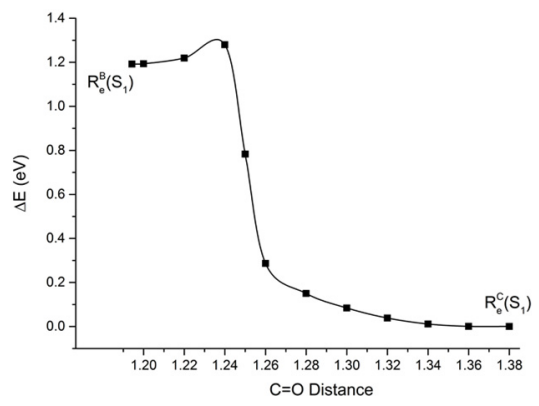

(b)

Figure 4. The geometry configuration of the global energy minimum of the APN's first excited state (a) and the PES of the transition state between the local minimum located at the benzene ring and the global equilibrium geometry of the APN's first excited state located at the carbonyl fragment (b).
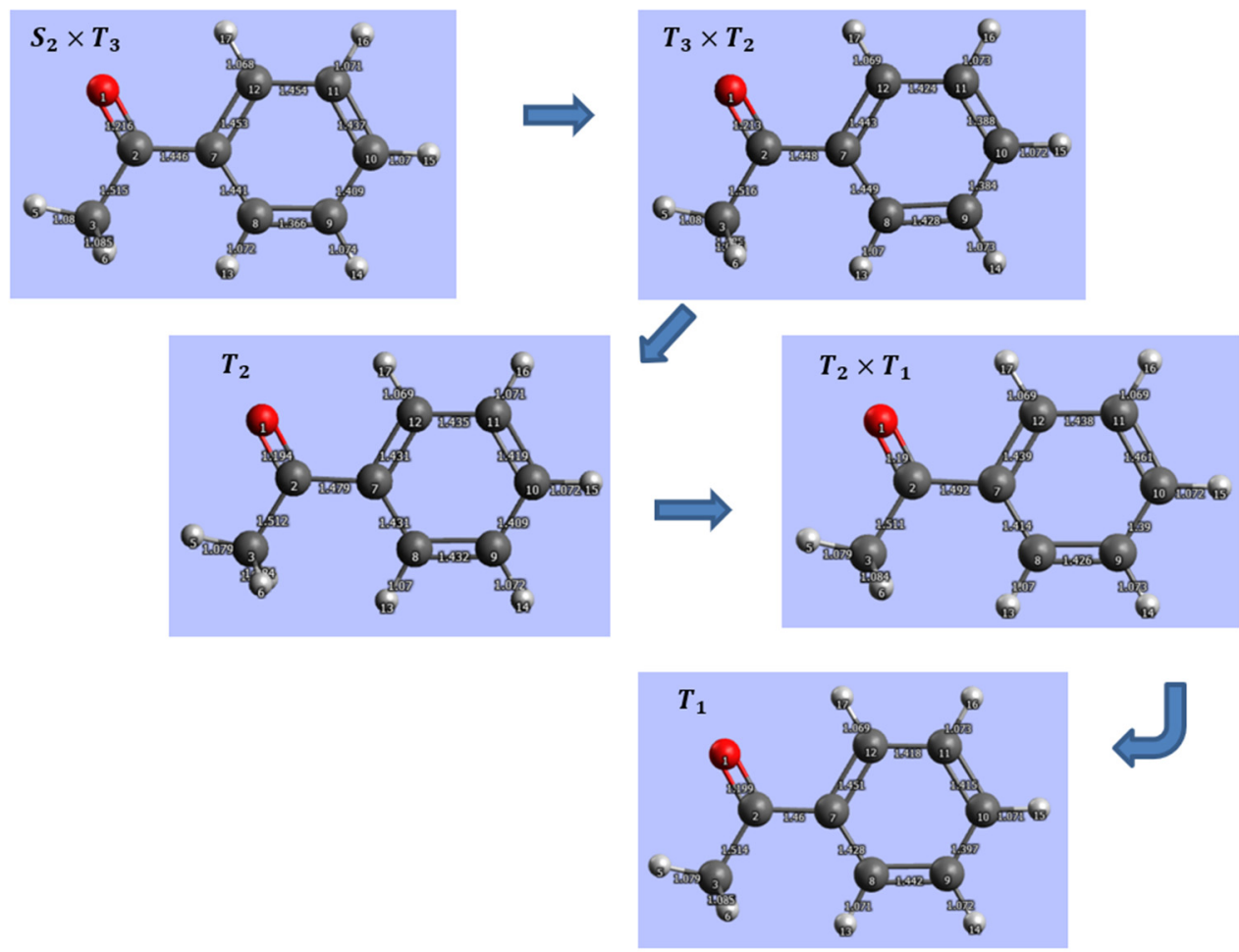

Figure 5. Geometry configurations of the critical points $\left(S_{2} \times T_{3} \rightarrow T_{3} \times T_{2} \rightarrow T_{2} \rightarrow\right.$ $T_{2} \times T_{1} \rightarrow T_{1}$ ) through the triplet relaxation pathway from the $S_{2} \times T_{3}$ intersystem crossing to the $T_{1}$ equilibrium geometry of APN molecule. 
In this case, the impediments could be the $0.63 \mathrm{eV}$ energy barrier and the constraint of the benzene ring's non-planar deformation (See Table 3). Calculations show further two conceivable scenarios, but they might happen

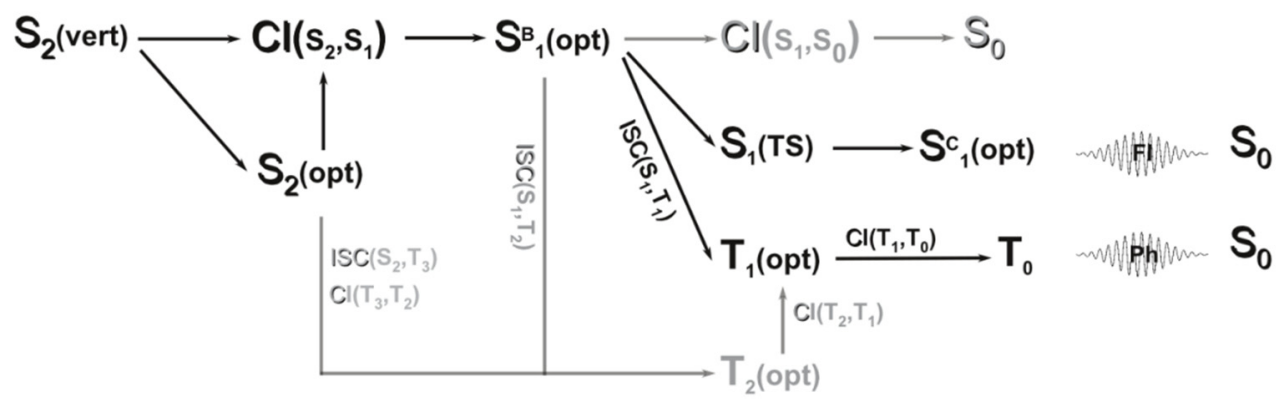

Figure 6. The possible relaxation pathways of the APN's low-lying electronic excited states.

with relatively small probabilities, due to the difficulty to achieve the different conditions necessary to reach the critical geometries. One of these deactivation schemes starts with the first step of the $S_{2}$ (vert) $\rightarrow S_{2}$ (opt) relaxation, but from the optimized $S_{2}$ singlet state the system directly switches to the $T_{3}$ triplet one, via the $S_{2} \otimes T_{3}$ ISC point. In this case the spinorbit coupling is lower almost two orders of magnitude $\left(0.32 \mathrm{~cm}^{-1}\right)$ than that for the $S_{1} \otimes T_{1}$ crossing, but practically the geometry can be reached without crossing any energy gap or getting large geometry distortions. The second relaxation channel shows common pathway until the $R_{e}^{B}\left(S_{1}\right)$ local energy minimum is reached, but instead of the $S_{1} \otimes T_{1}$ crossing it continues its relaxation passing the $S_{1} \otimes T_{2}$ ISC point and follows its excited state decay along the triplet relaxation channel. For this case the spin-orbit coupling has a reasonable value of $1.15 \mathrm{~cm}^{-1}$, but needs $0.6 \mathrm{eV}$ excess of energy to reach the critical geometry of the $S_{1} \otimes T_{2}$ ISC point. For the complete relaxation pathway see Figure 6.

\section{COMPUTATIONAL DETAILS}

The calculation of the equilibrium geometries as well as the localization of conical intersection $(\mathrm{Cl})$ and intersystem crossing (ISC) points between potential energy surfaces (PES) of the different electronic excited states of APN have been performed at the state-averaged CASSCF level of theory using the MOLPRO computational chemistry program package [17] In all cases the triple $\zeta$ quality def2-TZVP [18] basis set was 
taken and no symmetry restrictions were considered for molecular geometries. Four $\pi$ (one from $\mathrm{C}=\mathrm{O}$ group of the carbonyl fragment and three from the benzene aromatic ring) and one no lone pair orbital of the oxygen atom were taken as occupied orbitals in the active space for the APN case, which was further completed with four virtual orbitals. One should mention that, using four virtual orbitals instead of three, further oneelectron excitations are included in the excitation scheme and accordingly, better excitation energies can be obtained. In this way, the final electronorbital configuration of the active space for the APN becomes $(10,9)$, the nomenclature $(n, m)$ denoting here an active space of $n$ electrons and $m$ orbitals. In order to take into account, the effect of electron correlations (static and dynamic) for different excitation energies evaluation, we have performed further calculations using the extended multistate CASPT2 [19] method which provides a better description of near degenerate situations and avoided crossings. For this case of XMS-CASPT2 calculations a level shift [20] parameter of 0.2 was considered. The computation of individual spin-orbit matrix elements for a given pair of states were obtained considering the Breit-Pauli operator as described in ref.-s [21,22] and implemented in MOLPRO the program package. The charge distribution analysis was performed by natural population analysis (NPA) $[23,24]$ using the NBO module of the same MOLPRO package. The open source GABEDIT [25] molecular graphics program was used to visualize and analyze the molecular structures, and the molecular graphics (figures) were created using the GAUSSVIEW [26] molecule editor and visualizer software.

\section{CONCLUSIONS}

The full mapping of low-lying excited state relaxation dynamic pathways for acetophenone has been investigated considering the complete active space self-consistent field (CASSCF) and the extended multistate complete active space second-order perturbation theory (XMSCASPT2) methods. All critical geometry configurations, whether equilibrium or surface crossing point geometries, have been located on the potential energy hyper-surface and the strength of the spin-orbit coupling at the crossing point geometries were characterized. Results reveal several excited state deactivation pathways including both the singlet and triplet spin states, which lead either to radiative or non-radiative relaxation. In the case of radiative relaxation both the fluorescence and phosphorescence phenomena could occur, depending on which type of intersection points (conical intersection or intersystem crossing) the system passes through in its higher electron states. 
ATTILA BENDE

\section{ACKNOWLEDGMENTS}

This work was supported by the Ministry of Research and Innovation $\mathrm{MCl}$ Core Program, Projects IZO-MOL-EA Nr. PN19 3502 01. We gratefully acknowledge the Data Center of NIRDIMT Cluj-Napoca for providing computational infrastructure and the technical assistance.

\section{REFERENCES}

1. W. H. Fang; D. L. Phillips; ChemPhysChem, 2002, 3(10), 889-892.

2. J. Li; F. Zhang; W. H. Fang; J. Phys. Chem. A, 2005, 109(34), 7718-7724.

3. Q. Fang; Y. J. Liu; J. Phys. Chem. A, 2010, 114(1), 680-684.

4. M. Berger; C. Steel; J. Am. Chem. Soc., 1975, 97(17), 4817-4821.

5. R. D. Vanselow; A. B. F. Duncan; J. Am. Chem. Soc., 1953, 75(4), 829-832.

6. M. Koyanagi; R. J. Zwarich; L. J. Goodman; Chem. Phys., 1972, 56(6), 30443060.

7. Y.-W. Wang; H.-Y. He; W.-H. Fang; J. Mol. Struct. (THEOCHEM), 2003, 634(13), 281-287.

8. A. Bende; AIP Conf. Proc., 2013, 1565, 24-28.

9. M. Huix-Rotllant; D. Siri; N. Ferré; Phys. Chem. Chem. Phys., 2013, 15(44), 19293-19300.

10. M. Bear; Beyond Born--Oppenheimer: Electronic Nonadiabatic Coupling Terms and Conical Intersections, Wiley-Interscience, 2006.

11. H.-Y. Xiao; Y.-J. Liu; W.-H. Fang; J. Mol. Struct. (THEOCHEM), 2007, 802(1-3), 99-103.

12. G. Cui; Y. Lu; W. Thiel; Chem. Phys. Lett., 2012, 537, 21-26.

13. W.-H. Fang; Acc. Chem. Res., 2008, 41(3), 452-457.

14. M. Huix-Rotllant; N. Ferré; J. Chem. Phys., 2014, 140(13), 134305.

15. I. J. Palmer; I. N. Ragazos; F. Bernardi; M. Olivucci; M. A. Robb; J. Am. Chem. Soc., 1993, 115, 673-682.

16. Q. Li, D. Mendive-Tapia; M. J. Paterson; A. Migani; M. J. Bearpark; M. A. Robb; L. Blancafort; Chem. Phys., 2010, 377(1-3), 60-65.

17. MOLPRO version 2012.1 is a package of ab initio programs written by H.-J. Werner; P. J. Knowles; G. Knizia; F. R. Manby; M. Schütz; P. Celani; T. Korona; R. Lindh; A. Mitrushenkov; G. Rauhut; K. R. Shamasundar; T. B. Adler; R. D. Amos; A. Bernhardsson; A. Berning; D. L. Cooper; M. J. O. Deegan; A. J. Dobbyn; F. Eckert; E. Goll; C. Hampel; A. Hesselmann; G. Hetzer; T. Hrenar; G. Jansen; C. Köppl; Y. Liu; A. W. Lloyd; R. A. Mata; A. J. May; S. J.; McNicholas; W. Meyer; M. E. Mura; A. Nicklaß; D. P. O'Neill; P. Palmieri; D. Peng; K. Pflüger; R. Pitzer; M. Reiher; T. Shiozaki; H. Stoll; A. J. Stone; R. Tarroni; T. Thorsteinsson; M. Wang; see http://www.molpro.net.

18. F. Weigend; R. Ahlrichs; Phys. Chem. Chem. Phys., 2005, 7, 3297-3305. 
19. T. Shiozaki; W. Györffy; P. Celani; H.-J. Werner; J. Chem. Phys., 2014, 135(8), 081106.

20. J. Lorentzon; M. P. Fulscher; B. O. Roos; J. Am. Chem. Soc., 1995, 117(36), 9265-9273.

21. A. Berning; M. Schweizer; H.-J. Werner; P. J. Knowles; P. Palmieri; Mol. Phys., 2000, 98(21), 1823-1833.

22. A. O. Mitrushenkov; P. Palmieri; R. Tarroni; Mol. Phys., 2003, 101(13), 20432046.

23. J. P. Foster; F. Weinhold; J. Am. Chem. Soc., 1980, 102(24), 7211-7218.

24. A. E. Reed; R. B. Weinstock; F. Weinhold; J. Chem. Phys., 1985, 83(2), 735743.

25. A. R. Allouche; J. Comput. Chem., 2011, 32, 174-182.

26. GaussView, Version 5.0.9, R. Dennington, T. Keith, J. Millam, Semichem Inc., Shawnee Mission, KS, 2009. 
